\title{
Defined Criterion Group Option Relationship Priority Number
}

National Cancer Institute

\section{Source}

National Cancer Institute. Defined Criterion Group Option Relationship Priority Number. NCI Thesaurus. Code C93767.

An integer specifying the relative preference for considering this relationship before other similar relationships having the same source activity. 